\title{
Tumorerkrankungen bei Jugendlichen und jungen Erwachsenen (AYA) - Parallelen und Unterschiede zu jungen Erwachsenen mit einem angeborenen Herzfehler (EMAH)
}

Adolescents and Young Adults with Cancer - Analogies and Differences to Young Adults with Congenital Heart Defects

\section{T. Paul, R. Grabitz}

Bibliografie

DOI http://dx.doi.org/

$10.1055 / \mathrm{s}-0034-1371844$

Klin Padiatr 2014; 226: 51-52

(c) Georg Thieme Verlag KG

Stuttgart · New York

ISSN 0300-8630

Korrespondenzadresse

Prof. Dr. Thomas Paul

Abteilung für Pädiatrische Kardiologie und Intensivmedizin Universitätskinderklinik, Georg-August-Universität Robert-Koch-Straße 40 37075 Göttingen

Tel.: +49/551396203

Fax: $+49 / 5513922561$

tpaul@gwdg.de
Krebserkrankungen bei Jungendlichen und jungen Erwachsenen zeichnen sich durch biologische Besonderheiten aus. Fortschritte in der Therapie von Tumorerkrankungen bei Jugendlichen und jungen Erwachsenen sind abhängig vom Verständnis des biologischen Verhaltens der Erkrankungen sowie auch der besonderen Bedürfnisse dieser Altersgruppe. Aufgrund dieser besonderen Situation sind spezialisierte Behandlungseinheiten für diese Patienten etabliert worden mit dem Ziel das Überleben und die Lebensqualität dieser Patientengruppe zu verbessern [6]. Dies erfordert unter anderem die bestehenden Strukturen zu verbessern für eine integrierte Zusammenarbeit von Kinderonkologen und internistischen Onkologen mit einem freien $\mathrm{Zu}$ gang zu der erforderlichen Expertise für das gesamte multidisziplinäre Team ohne eine Begrenzung durch die bestehenden traditionellen $\mathrm{Ge}$ sundheitsstrukturen. Zudem bedarf es der Schaffung einer Umgebung, welche den speziellen Bedürfnissen der Patienten gerecht wird. Es besteht der Bedarf für spezielle Behandlungsprotokolle für diese Patientengruppe.

Im Jahr 2011 wurde im Universitätsklinikum Halle eine Behandlungseinheit für Jugendliche und junge Erwachsene (adolescents and young adults, AYA) im Alter von 15 bis 25 Jahren mit einer neu diagnostizierten Tumorerkrankung gegründet. Erste Erfahrungen zeigen eine hohe $\mathrm{Zu}$ friedenheit über die neuartige Behandlungsstruktur bei den Patienten und den Familien [2]. Weitere Schritte sind die Erarbeitung eines Curriculums für die Ausbildung aller beteiligten Berufsgruppen sowie die Fokussierung auf das biologische Verhalten der Tumorerkrankungen und die potentiellen therapiebedingten Spätfolgen im Zusammenhang mit den psychosozialen Besonderheiten dieser Altersgruppe. Hierauf basierend sollte eine optimale Behandlungsstrategie entwickelt werden.

Durch diese gemeinsamen Aktivitäten der pädiatrischen sowie der internistischen Onkologen ist in den letzen Jahren eine Verbesserung der Versorgungsstrukturen und der medizinischen Versorgung für Patienten dieser Altersgruppe erzielt worden.

Bei Jugendlichen und jungen Erwachsenen mit einem angeborenen Herzfehler (EMAH) stellt sich eine grundsätzlich andere Situation und Herausforderung an die behandelnden Ärzte dar. Durch die Fortschritte der Kinderkardiologie und Kinderherzchirurgie erreichen heute ca. 90\% aller Patienten mit einem angeborenen Herzfehler das Erwachsenenalter. Die Gesamtzahl der Er- wachsenen mit einem angeborenen Herzfehler liegt derzeit in Deutschland bei mehr als 250000 mit einem beträchtlichen Anteil von Patienten von bis zu 50\% mit einem komplexen Herzfehler. Diese Patientengruppe stellt eine enorme Herausforderung an die internistischen Kardiologen wie auch die Kinderkardiologen dar. Überwiegen bei Jugendlichen noch die speziellen Aspekte des angeborenen Herzfehlers mit den residuellen hämodynamischen und elektrophysiologischen Residuen, so kommen mit zunehmendem Alter dieser Patienten die Probleme der erworbenen Herz- und Kreislauferkrankungen wie die arterielle Hypertension, die koronare Herzerkrankung sowie die Herzinsuffizienz hinzu. Die spezifischen Probleme dieser Altersgruppe bei den verschiedenen Herzfehlern sind von den internationalen wie auch nationalen Fachgesellschaften in Konsensuspapieren zusammengestellt worden $[1,5,7]$. Von den Fachgesellschaften wurden durch gemeinsame Arbeitsgruppen Empfehlungen für die Struktur der Versorgung sowie für die Qualifikation der Ärzte erarbeitet, welche die Versorgung der Erwachsenen mit einem angeborenen Herzfehler durchführen [3,4]. Die Mehrzahl der Erwachsenen mit einem angeborenen Herzfehler wird von Hausärzten oder Internisten betreut, je nach Komplexität des Herzfehlers wird eine ambulante Mitbetreuung durch zertifizierte regionale EMAH-Schwerpunktpraxen bzw. EMAH-Schwerpunktkliniken empfohlen. Patienten mit einem angeborenen Herzfehler mit mittlerer und hoher Komplexität sollen durch ein überregionales EMAH-Zentren versorgt werden. Die Versorgung von Jugendlichen und Erwachsenen mit einer Krebserkrankung unterscheidet sich erheblich von der Versorgung von EMAHPatienten. Bei der ersten Patientengruppe liegt eine akut erworbene lebensbedrohliche Erkrankung vor, bei den EMAH-Patienten steht die Transition dieser Patienten mit einer angeborenen chronischen Erkrankung aus der kinderkardiologischen Versorgung in die Erwachsenenmedizin im Vordergrund. Analogien bestehen in der psychosozialen Bedürfnissen diese Patientengruppe, auf welche die Pädiater mit absoluter Selbstverständlichkeit eingehen. Für beide Patientengruppen gemeinsam ist die enge Kooperation zwischen den pädiatrischen und internistischen Kollegen entscheidend für eine optimale medizinische Versorgung. Die gemeinsamen Arbeitsgruppen der Deutschen Gesellschaft für Pädiatrische Kardiologie und der Deutschen Gesellschaft für Kardiologie haben die Struktur- und Weiterbildungsvoraussetzungen für die Versorgung der 
EMAH-Patienten definiert [3, 4]. Diese können als Grundlage für die Strukturierung der Versorgung von Jugendlichen und jungen Erwachsenen mit Krebserkrankungen dienen.

\section{Literatur}

1 Baumgartner H, Bonhoeffer P, De Groot NM et al. Task Force on the Management of Grown-up Congenital Heart Disease of the European Society of Cardiology (ESC); Association for European Paediatric Cardiology (AEPC); ESC Committee for Practice Guidelines (CPG) (2010) ESC Guidelines for the management of grown-up congenital heart disease (new version). Eur Heart J 2010; 31: 2915-2957

2 Bernig T, Jahn F, Witkowsky S et al. Treatment of adolescents and young adults (AYA) with cancer in a multidisciplinary setting: on the way to a highly specialized AYA unit. Klin Padiatr 2013; 225: 335-338

3 Hess J, Bauer U, de Haan F et al. Recommendations for adult and paediatric cardiologists on obtaining additional qualification in "Adults with Congenital Heart Disease" (ACHD). Int J Cardiol 2011; 149: 186-191

4 Kaemmerer H, Breithardt G. Kommission für Klinische Kardiologie der Deutschen Gesellschaft für Kardiologie Empfehlungen zur Qualitätsverbesserung der interdisziplinären Versorgung von Erwachsenen mit angeborenem Herzfehler (EMAH). Clin Res Cardiol 2006; 95 (Suppl 4): $76-84$
5 Schmaltz AA, Bauer U, Baumgartner $H$ et al. Medizinische Leitlinie zur Behandlung von Erwachsenen mit angeborenen Herzfehlern (EMAH) der deutsch-östereichisch-schweizerischen Fachgesellschaften. Clin Res Cardiol 2008; 97: 194-214

6 Stark D, Lewis I. Improving outcomes for teenagers and young adults (TYA) with cancer. Klin Padiatr 2013; 225: 331-333

7 Warnes CA, Williams RG, Bashore TM et al. American College of Cardiology; American Heart Association Task Force on Practice Guidelines (Writing Committee to Develop Guidelines on the Management of Adults With Congenital Heart Disease); American Society of Echocardiography; Heart Rhythm Society; International Society for Adult Congenital Heart Disease; Society for Cardiovascular Angiography and Interventions; Society of Thoracic Surgeons. ACC/AHA 2008 guidelines for the management of adults with congenital heart disease: a report of the American College of Cardiology/American Heart Association Task Force on Practice Guidelines (Writing Committee to Develop Guidelines on the Management of Adults With Congenital Heart Disease). Developed in collaboration with the American Society of Echocardiography, Heart Rhythm Society, International Society for Adult Congenital Heart Disease, Society for Cardiovascular Angiography and Interventions, and Society of Thoracic Surgeons. J Am Coll Cardiol 2008; 52: 143-263 